\title{
Distal and lateral toenail onychomycosis caused by Trichophyton rubrum: treatment with photodynamic therapy based on methylene blue dye
}

\author{
Linton Wallis Figueiredo Souza
}

Simone Vilas Trancoso Souza ${ }^{2}$

Ana Cristina de Carvalho Botelho ${ }^{3}$

DOI: http://dx.doi.org/10.1590/abd1806-4841.20142197

\begin{abstract}
The study showed the effectiveness of photodynamic therapy based on methylene blue to treat severe distal and lateral subungual toenail onychomycosis. 22 patients were divided into two groups: group A consisting of 11 patients with severe toenail onychomycosis and group B consisting of 11 patients with mild-to-moderate toenail onychomycosis. All patients had onychomycosis caused by Trichophyton rubrum. The patients were treated with sessions of $2 \%$ methylene blue aqueous solution irradiated with light emission diode device with $630 \mathrm{~nm}$ and $36 \mathrm{~J} / \mathrm{cm} 2$ biweekly for six months. The clinical response was significantly better in patients with mild-to-moderate $(100 \%)$ onychomycosis compared with patients with severe onychomycosis $(63.6 \%)$.
\end{abstract}

Keywords: Nail diseases; Phototherapy; Trichophyton

Severe distal-lateral subungual onychomycosis (SDSO) is characterized by fungal invasion of the hyponychium through the distal subungual and lateral nail groove reaching the lunula or nail matrix, or by a $50 \%$ nail plate involvement associated with $>2 \mathrm{~mm}$ subungual hyperkeratosis or dermatophytoma. ${ }^{1,2}$ Patients with SDSO are categorized as poor responders or nonresponders to systemic antifungal treatment and are difficult to treat. ${ }^{3}$ In severe onychomycosis, the nail surface is likely to retain an abnormal appearance even after mycological cure. ${ }^{4}$ The first-line systemic drugs itraconazole and terbinafine promote complete healing in $25 \%$ and $35 \%$ of severe cases, respectively., Therefore the current best treatment option is the use of combination antifungal therapy (systemic + topical + nail avulsion or abrasion) with supplementation, after 3-6 months drug-free. ${ }^{4}$ However, a need remains for more effective oral antifungal agents with fewer adverse effects and fewer drug-drug interactions, as well as for more effective topical agents with greater nail penetration. ${ }^{3,4}$
Photodynamic therapy (PDT) is a medical modality that combines the use of visible light and a photosensitive compound in the presence of oxygen. It is widely used to treat nonmelanoma skin cancer. ${ }^{5}$ PDT is a noninvasive treatment for onychomycosis and can selectively destroy infectious pathogens. ${ }^{6}$ Methylene blue (MB) and other dyes of the same class exhibit intense absorption at the 600-660-nm red light region. ${ }^{3}$ Previous studies have demonstrated that MB-light emission diode (MBLED)/PDT is effective and safe, with response rates of approximately $85-100 \%, 5,7$ We here investigated the efficacy of MBLED/PDT in patients with toenail SDSO caused by Trichophyton rubrum.

Our open-label controlled clinical trial included 11 immunocompetent patients with toenail SDSO and 11 immunocompetent patients with mild-to-moderate distal and lateral subungual toenail onychomycosis (MDSO) with clinical and mycological diagnosis. The inclusion criteria were clinical signs of onychomycosis confirmed by direct microscopic examination of nail material with $20 \%$ potassium hydroxide, and by cul-

Received on 14.10.2012.

Approved by the Advisory Board and accepted for publication on 31.10.2012.

* Work performed at the Clemente Faria University Hospital - Montes Claros State University (HUCF - UNIMONTES) - Montes Claros (MG), Brazil. Conflict of interest: None

Financial Support: None

Master in Health Sciences - Professor of Dermatology and Research Professor, Department of Clinical Medicine, Center for Infectious Diseases Research Clemente Faria University Hospital - Montes Claros State University (HUCF - UNIMONTES) - Montes Claros (MG), Brazil.

Specialist, student in the Postgraduate Program in Health Sciences and Designated Professor - Montes Claros State University (UNIMONTES) - Montes Claros (MG), Brazil

Post-Doctorate in Parasitology - Professor in the Health Sciences Postgraduate Program of the Montes Claros State University (UNIMONTES) - Montes Claros (MG), Brazil. 
ture on Sabouraud's agar with chloramphenicol and cycloheximide and Sabouraud's agar with chloramphenicol. None of the patients showed nail changes associated with skin or systemic diseases, nor had used antifungal medications in the previous 4 months. PDT consisted of 6 months of MBLED sessions with a 15-day interval between sessions. A $2 \%$ aqueous $\mathrm{MB}$ solution was applied to the lesion followed by irradiation with red light $\left(630 \mathrm{~nm}, 36 \mathrm{~J} / \mathrm{cm}^{2}\right)$ from an LED device with a light intensity output of $3100 \mathrm{~mW} / \mathrm{cm}^{2}$ and optical intensity of $100 \mathrm{~mW} / \mathrm{cm}^{2}$ (Multiwaves; Industra, São Carlos, Brazil). Patients with hyperkeratotic lesions or dermatophytomas were treated with a rotation abrasive device with a 3-mm diamond tip (Dermoabrasor; Bley Med, Curitiba, Brazil). Patients were evaluated monthly during the treatment period. The microbiological and clinical outcomes were assessed at the end of treatment. The data were described using means (standard deviations), proportions and constructing 95\% confidence intervals (CI). This study was approved by the ethics committee of the Montes Claros State University.

Before treatment, all 22 patients were found positive for T. rubrum on mycological examination. Figure 1 shows the stain delimiting the impaired area of the nail plate toward the matrix and the area of subungual hyperkeratosis. The dye did not penetrate the area with fungal biofilm in the nail plate. This noncolored area was subjected to dermabrasion. The nail plate detached from the nail bed during the healing process because of nail matrix injury. All patients showed clinical improvement with mycological cure (Table 1). The clinical response rate at last visit was significantly better in patients with MDSO (100\%; CI $100 \%)$ compared with patients with SDSO $(63.3 \%$; CI $35-92 \%)$. No adverse effects were observed.

This study shows the effectiveness of MBLED/PDT for the treatment of SDSO caused by $T$. rubrum. SDSO is the major cause of treatment failure in patients with onychomycosis. ${ }^{3}$ Studies on combined topical and systemic therapies for SDSO reported a mycological cure rate of between $27 \%$ and $83 \% .{ }^{4}$ PDT with MB has been used for several decades to treat cutaneous fungal infections. ${ }^{5}$ In this study, $\mathrm{MB}$ showed excellent penetration into the nail plate in all patients, including those with nail matrix impairment, unlike other topical antifungals. ${ }^{4}$ Another advantage of MBLED/PDT over other treatments is the possibility of delimiting areas with fungal biofilms, found in dermatophytomas and fungal longitudinal streaks, which are determinants of treatment failure. ${ }^{3}$ Previous clinical trials and in vitro studies have demonstrated the effectiveness of PDT, but indicated the need for an adequate energy density and concentration of the photosensitive dye., ${ }^{5,7}$ Another study showed that the fungicidal activity of PDT can be enhanced by increasing the energy density, which might reduce the number of sessions and the treatment duration. ${ }^{8}$ The use of macrocyclic molecules with fewer sessions ( 3 on average), because of the high cost, has resulted in a clinical
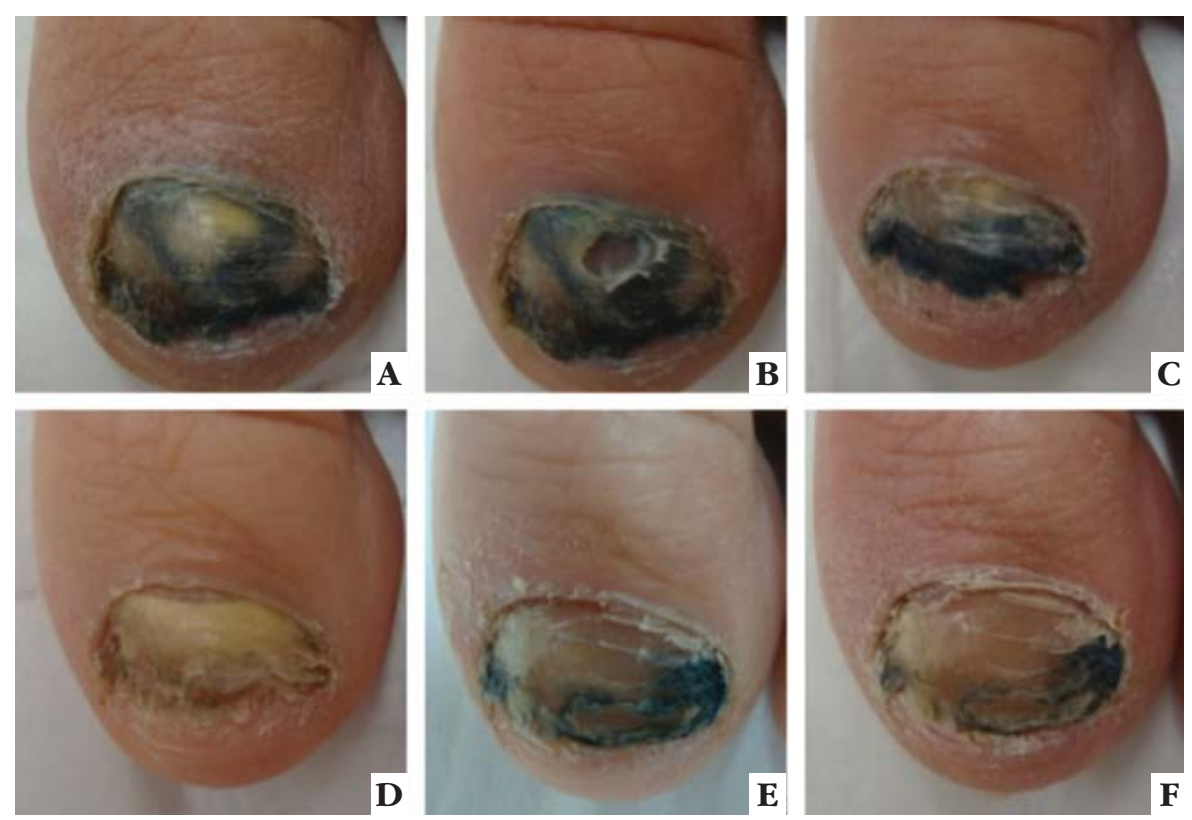

FIGURE 1: Severe distal and lateral toenail onychomycosis treated with photodynamic therapy based on methylene blue dye. A before treatment; B nail abrasion; C 2 months; D 3 months; E 5 months; $\mathbf{F} 6$ months. 
TABLE 1: Baseline demographics, disease characteristics and clinical response in 22 patients with distal and lateral toenail onychomycosis treated with photodynamic therapy.

\begin{tabular}{llll}
\hline & & Group A $(\%)^{*} \mathbf{n}=\mathbf{1 1}$ & Group B (\%) $)^{* *} \mathbf{n}=\mathbf{1 1}$ \\
\hline $\begin{array}{l}\text { Gender } \\
\text { Female }\end{array}$ & & $4(36.4)$ & $6(54.5)$ \\
Male & & $5(63.6)$ & $5(45.5)$ \\
Age, years, mean + SD & & $54.2+10.8$ & $48.8+8.4$ \\
Duration, year, mean + SD & & $4.5+2.1$ & $3.8+1.7$ \\
Clinical response*** - & weeks & & \\
Complete & 12 & - & $4(36.4)$ \\
& 24 & $6(54.5)$ & $11(100)$ \\
Marked & 48 & $7(63.6)$ & $7(63.6)$ \\
& 12 & $3(27.3)$ & - \\
Moderate & 24 & $4(45.5)$ & - \\
& 48 & $3(27.3)$ & - \\
Mild & 12 & - & - \\
& 24 & - & - \\
\hline
\end{tabular}

Group A= severe distal and lateral toenail onychomycosis; Group B= Mild / moderate distal and lateral toenail onychomycosis; *Severe grading (lunula or matrix involvement, or $>50 \%$ nail involvement with dermatophytoma or subungual hyperkeratosis $>2 \mathrm{~mm}$ ); **Mild / moderate grading $(<75 \%$ nail involvement or $<50 \%$ nail involvement with dermatophytoma or subungual hyperkeratosis $>2 \mathrm{~mm})$; ${ }^{* * *}$ Clinical response during treatment for 6 months and follow-up: complete $(100 \%)$; marked ( $\left.>75 \%\right)$, moderate (50-75\%), Mild $(<50 \%)$.

cure rate lower than that of azole drugs, probably due to the inadequate number of sessions. ${ }^{9,10}$

The results of this study confirm that MBLED/PDT is safe and effective, with a favorable outcome in the treatment of SDSO caused by $T$. rubrum. Finally, MB facilitates the diagnosis of the

\section{REFERENCES}

1. Baran R, de Berker D, Holzberg M, Thomas L. Baran \& Dawber Disease of the nails and their management. 4th ed. Chichester: Wiley-Blackwell; 2012.

2. Carney C, Tosti A, Daniel R, Scher R, Rich P, DeCoster J, et al. A new classification system for grading the severity of onychomycosis: Onychomycosis Severity Index. Arch Dermatol. 2011;147:1277-82.

3. Scher RK, Baran R. Onychomycosis in clinical practice: factors contributing to recurrence. Br J Dermatol. 2003;149:5-9.

4. Singal A, Khanna D. Onychomycosis: Diagnosis and management. Indian J Dermatol Venereol Leprol. 2011;77:659-72.

5. Tardivo JP, Del Giglio A, Oliveira CS, Gabrielli DS, Junqueira HC, Tada DB, Severino D. Methylene blue in photodynamic therapy: From basic mechanisms to clinical applications. Photodiagnosis Photodyn Ther. 2005;2:175-91.

6. Zeina B, Greenman J, Corry D, Purcell WM. Antimicrobial photodynamic therapy: assessment of genotoxic effects on keratinocytes in vitro. $\mathrm{Br} J$ Dermatol. 2003:148:229-32.

7. Scwingel AR, Barcessat AR, Núñez SC, Ribeiro MS. Antimicrobial photodynamic therapy in the treatment of oral candidiasis in HIV-infected patients. Photomed Laser Surg. 2012;30:429-32.

8. Amorim JC, Soares BM, Alves OA, Ferreira MV, Sousa GR, Silveira Lde B, et al. Phototoxic action of light emitting diode in the in vitro viability of Trichophyton rubrum. An Bras Dermatol. 2012;87:250-5. presence of a fungal biofilm in the nail plate and nail bed. Further clinical studies may indicate the optimal energy density needed to improve the therapeutic outcome, and its possible associations with systemic treatments.
9. Smijs TG, Pavel S. The susceptibility of dermatophytes to photodynamic treatment with special focus on Trichophyton rubrum. Photochem Photobiol. 2011;87:2-13.

10. Sotiriou E, Koussidou-Eremonti T, Chaidemenos G, Apalla Z, Ioannides D. Photodynamic therapy for distal and lateral subungual toenail onychomycosis caused by Trichophyton rubrum: Preliminary results of a single-centre open trial. Acta Derm Venereol. 2010;90:216-7.
MAILING ADDRESS:
Linton Wallis Figueiredo Souza
Avenida Cula Mangabeira, 562
Sto Expedito
39401-001 Montes Claros (MG)
Brazil
E-mail:wallis@uai.com.br

How to cite this article: Souza LWF, SouzaSVT, Botelho ACC. Distal and lateral toenail onychomycosis caused by Trichophyton rubrum: treatment with photodynamic therapy based on methylene blue dye. An Bras Dermatol. 2014;89(1):184-6. 